\title{
Successful treatment of tracheoesophageal fistula using a covered esophageal stent
}

Jung Hun Kim and Young Koog Cheon

Department of Internal Medicine, Konkuk University Medical Center, Seoul, Korea
Received: October 11, 2017

Revised : February 12, 2018

Accepted: February 14, 2018

\section{Correspondence to}

Young Koog Cheon, M.D.

Tel: +82-2-2030-7490

Fax: +82-2-2030-7748

E-mail: ykskyoo1@hanmail.net
We report herein a case of superficial esophageal cancer not eligible for endoscopic submucosal dissection. This patient developed a tracheo-esophageal fistula after radiation therapy followed by photodynamic therapy (PDT).

A 74-year-old male underwent total gastrectomy due to advanced gastric cancer in 2014. In 2017, a flat discolored lesion encircling the lumen at 30 to $35 \mathrm{~cm}$ from the incisors was found by gastroscopy. Lugol's solution showed an unstained mucosal lesion (Fig. 1A). Radiotherapy was performed at a different hospital, because the patient did not consent to surgery. Follow-up gastroscopy showed squamous cancer at the radiotherapy site. The patient was transferred to our hospital for PDT, which was performed four times using a 2-cm light diffuser and $160 \mathrm{~J} / \mathrm{cm}$. The
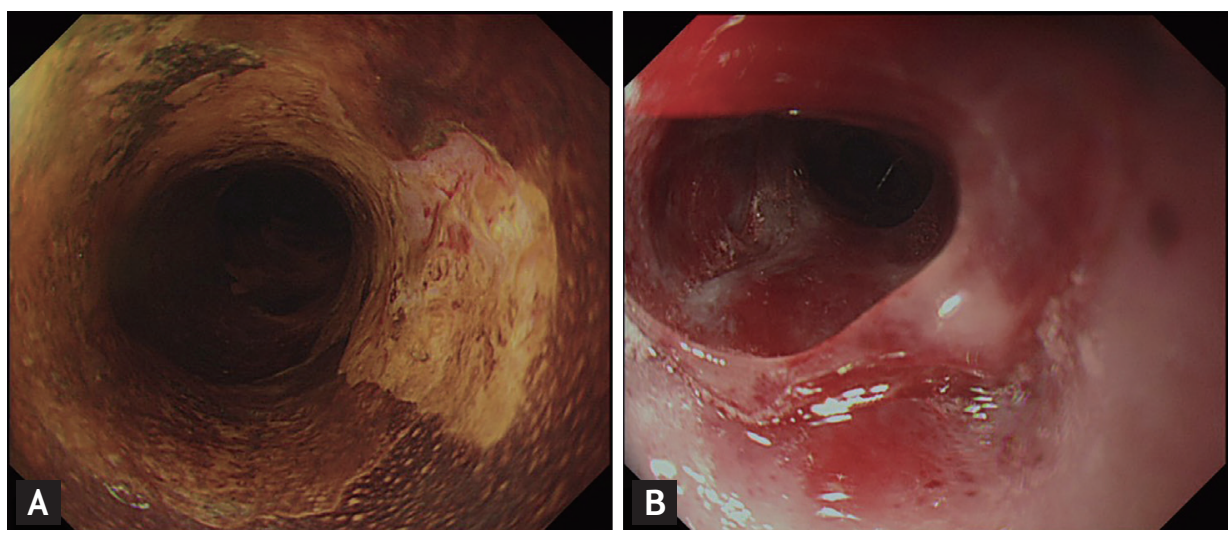

Figure 1. (A) Staining with Lugol's solution. (B) Post-photodynamic therapy image. 



Figure 2. (A) Endoscopic view of the transesophageal fistula. A fully covered metal esophageal stent was inserted on June 30 . (B) Bronchoscopic view of the transesophageal fistula in the distal trachea. The membrane of the stent is intact.

esophageal fistula that developed after PDT by insertion of a covered esophageal stent.

Written informed consents were obtained.

\section{Conflict of interest}

No potential conflict of interest relevant to this article was reported. 